\title{
Probing the Heart of an Active Galactic Nucleus: NGC 1068
}

\author{
Matthias Tecza, Niranjan Thatte \\ Max-Planck-Instutit für extraterrestrische Physik, Postbox 1312, \\ D-85741 Garching, Germany \\ Roberto Maiolino \\ Osservatorio Astrofisico di Arcetri, Largo E. Fermi, Firenze, Italy
}

\begin{abstract}
We present results from integral field spectroscopy of the narrow line region of NGC 1068, carried out with the MPE 3D near infrared imaging spectrometer. A map and velocity field of the [Fe II] fine structure line at $1.64 \mu \mathrm{m}$ is presented. The kinematics of the [Fe II] emission, which arises in partially ionized zones, shows red shifted emission in the north eastern cone of the NLR, and blue shifted emission in the south west, reversed relative to high ionization species such as [Si VI] or [O III]. We propose a model geometry of the narrow line region which is consistent with existing data and explains the observed [Fe II] kinematics.
\end{abstract}

\section{Introduction}

The narrow line region (NLR) of the Seyfert 2 galaxy NGC 1068 has been extensively studied at wavelengths ranging from the X-rays (Netzer and Turner 1997) to the radio (Wilson and Ulvestad 1983). Integral field spectroscopy of the near infrared [Fe II] line, which arises in partially ionized regions, allows us to study, for the first time, the spatial morphology of low excitation lines and draw conclusions about their excitation mechanisms.

\section{The [Fe II] emission}

The [Fe II] $1.64 \mu \mathrm{m}$ emission line $\left(\mathrm{a}^{4} \mathrm{~F}_{9 / 2}-\mathrm{a}^{4} \mathrm{D}_{7 / 2}\right)$ is the brightest emission feature in the $\mathrm{H}$ band long slit nuclear spectrum of NGC 1068 (Moorwood and Oliva 1993). The ionization potential of the iron atom $\left(\mathrm{Fe} \rightarrow \mathrm{Fe}^{+}\right)$is $7.87 \mathrm{eV}$, and the emission predominantly emanates from partially ionized regions in active galactic nuclei (Mouri et al. 1990). Figure 1 shows the integrated emission in the nuclear region of NGC 1068, as observed with the MPE 3D spectrometer. The emission morphology is roughly conical, with the apex south-west of the active nucleus, and a rather abrupt termination of the emission $\approx 3$ '. 2 north-east of the nucleus. The emission peak is located $0^{\prime \prime} \cdot 91$ north and $0^{\prime \prime} .32$ east of the $\mathrm{K}$ band continuum peak. The opening angle of the observed cone is $55^{\circ}$, consistent with that observed for the [O III] emission by Macchetto et al. (1994). However, 
the authors do point out that the axis of symmetry and the location of the apex of the cone depend on the intensity level at which the maps are presented. An additional effect in our data is the blurring of the cone boundaries due to seeing effects.

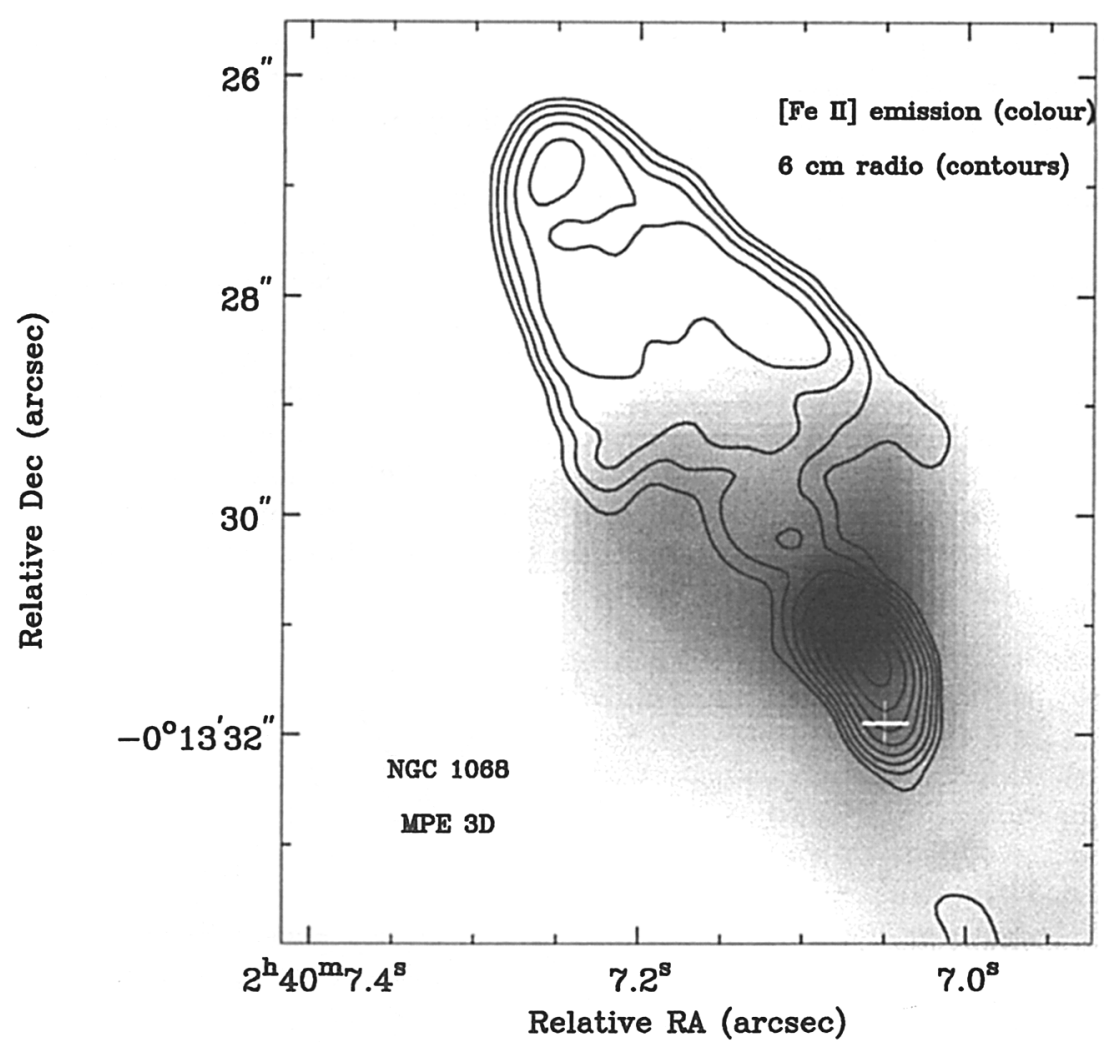

Figure 1. Integrated emission line map of the [Fe II] emission in the NLR of NGC 1068 (grayscale) with the $6 \mathrm{~cm}$ radio continuum emission (Wilson and Ulvestad 1983) overlaid in contours. An almost perfect anti-correlation is observed.

There is good overall agreement with the emission line map of Blietz et al. (1994), however the very low level extensions seen by Blietz et al. are not present in our map, especially the extension into the northern lobe of the jet roughly 2.5 from the emission peak.

The kinematics of the [Fe II] emission is very surprising, as its kinematic profile is completely reversed relative to all other emission lines in NGC 1068. The peak of the emission profile is red shifted for all apertures in the northeastern NLR, and blue shifted in the south west. The redshift increases with increasing distance north east of the active nucleus, a trend similar to that observed in the increasing blue shift of the [Si vI] line, but with a much smaller amplitude. 


\section{Kinematic model of the NLR}

Figure 2 illustrates our kinematic model of the NLR of NGC 1068. It shows some of the key features of the NLR as they would appear if viewed along a line of sight in the plane of the galaxy which is at right angles to our viewing direction. The north-east part of the ionization cone as seen by us is in the upper right of the figure, and the south-west part is in the lower left. The thin horizontal lines schematically represent the plane of the galaxy with a scale height of a few hundred pc. The central engine lies at the center of the figure. The dot-dashed lines delineate the ionization double-cone. The opening angle of the north eastern cone is $\approx 55^{\circ}$ (Macchetto et al. 1994). The heavy solid line represents the radio jet, which bends sharply on the NE side (Gallimore et al. 1996). The jet axis is inclined $\approx 70^{\circ}$ to our line of sight. The dashed curves symbolize the boundaries of the radio lobes where the expanding bow shock generated by the jet is interacting with quiescent interstellar material (Axon et al. 1998). The boundary is more pronounced where the bow shock encounters denser material closer to the plane of the galaxy (labelled "low ionization lines"). The other side (labelled "high ionization lines") sees more rarified material away from the galactic plane. The gas within the radio lobes shares the expansion velocity of the lobe, as depicted by the arrows.

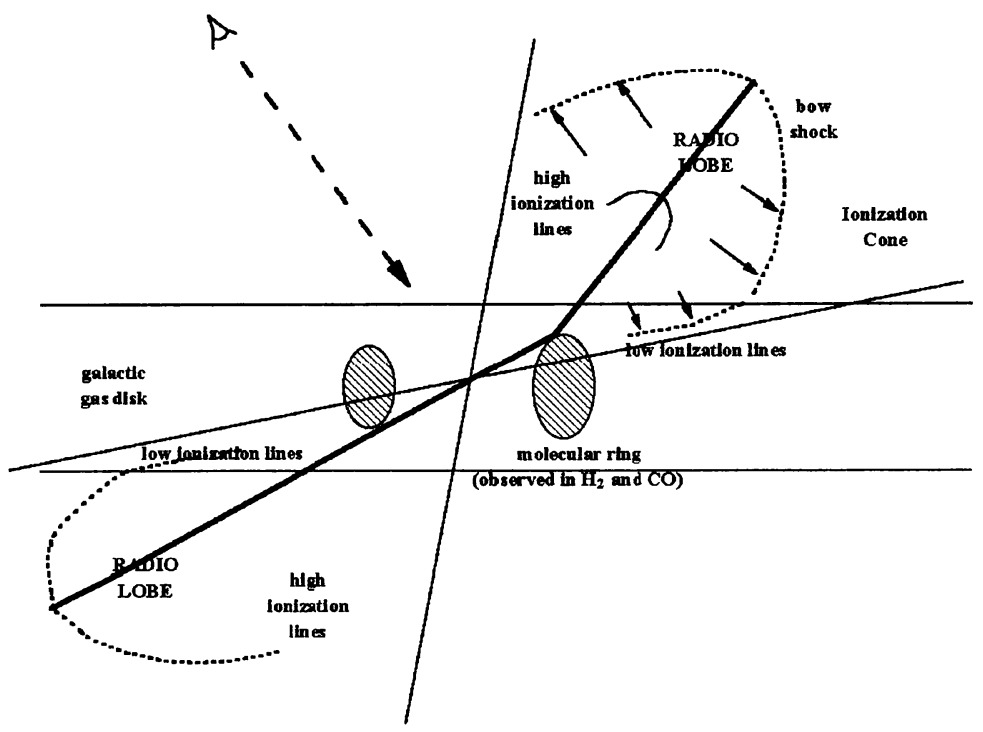

Figure 2. Proposed kinematic model of the NLR of NGC 1068.

We interpret the emission from high ionization species as orginating predominantly from clouds in rarified region of the north eastern NLR. These clouds are exposed to the intrinsic continuum from the central ionizing source. Our radiative transfer calculations show that very little [Fe II] or [O I] emission is produced from this region, since almost all of the iron and oxygen is in higher ionization states. The radio lobe is expanding fastest closest to the jet working surface, causing the observed blue shift of the high ionization species to increase with increasing distance from the active nucleus. 
The part of the north eastern radio lobe toward the galactic disk of NGC 1068 encounters dense, quiescent galactic interstellar medium gas. As the bow shock runs into denser, neutral material, it destroys most of the dust, enhancing the gas phase iron abundance. If we assume galactic ISM abundances for the pre-shock gas, and solar abundances for the post-shock gas, the expected enhancement in iron abundance is a factor of 50 . The post-shock gas experiences an attenuated ionizing radiation field, since it does not have a direct view of the active nucleus. Kraemer, Ruiz and Crenshaw (1998) have postulated that (part of) the NLR of NGC 1068 is partially shielded from the ionizing radiation of the active nucleus. Our photoionization computations show that an absorber with a total gas column density of $\mathrm{N}_{\mathrm{H}} \approx 10^{20} \mathrm{~cm}^{-2}$ can produce the required attenuation. While such an attenuator can effectively consume all photons from the central engine near the hydrogen ionzation edge, higher energy photons can penetrate through the attenuating column, creating a large partially ionized zone. Almost all the observed [Fe II] emission comes from this partially ionized region, which participates in the expansion driven by the bow shock, appearing redshifted to us.

\section{Conclusion}

Our kinematic model implies that the dominant fraction of the [Fe II] emission is produced in the regions where the expanding radio lobes intersect the plane of the galactic disk in NGC 1068. The observed [Fe II] emission comes from shocked disk gas which has an enhanced abundance of gas phase iron due to dust destruction by the shock. The emission appears to be excited by high energy photons from the central engine, as the observed spatial extent of the [Fe II] emission perfectly follows the ionization cone of higher ionization species. The [FeII] emitting gas is partially shielded from the ionizing continuum radiated by the central engine, creating an extended partially ionized zone. Our conclusions are strengthened by the observed perfect anti-correlation between the [Fe II] emission and the radio continuum emission (Fig 1.), and by the observed kinematics and line ratios of other species, deduced from archival HST FOS spectra.

\section{References}

Axon, D. J., Marconi, A., Capetti, A., Macchetto, F. D., Schreier, E., and Robinson, A. 1998, ApJ, 496, L75.

Blietz, M. et al. 1994, ApJ, 421, 92.

Gallimore, J. F., Baum, S. A., O'Dea, C. P. and Pedlar, A. 1996, ApJ, 458, 136.

Kraemer, S. B., and Crenshaw, D. M. 2000, ApJ, 532, 256.

Kraemer, S. B., Ruiz, J. R., and Crenshaw, D. M. 1998, ApJ, 508, 232.

Macchetto, F., Capetti, A., Sparks, W. B., Axon, D. J., and Boksenberg, A. 1994, ApJ, 435, L15.

Moorwood, A. F. M., and Oliva, E. 1993, A.S.P. Conf. Series, 41, 305.

Mouri, H., Nishida, M., Taniguchi, Y., and Kawara, K. 1990, ApJ, 360, 55.

Wilson, A., and Ulvestad, J. 1983, ApJ, 275, 8. 